\title{
REGIOSELECTIVE SYNTHESIS OF 2-SUBSTITUTED BENZOTHIAZOLOQUINOLATES AND THEIR BIOLOGICAL EVALUATION
}

\author{
EVS. Subrahmanyam*, A. Sravanthi and AR. Shabaraya \\ Department of Pharmaceutical Chemistry, Srinivas College of Pharmacy, \\ Mangalore, Karnataka-574143, India.
}

\begin{abstract}
Benzothiazoles containing nitrogen, sulphur as heteroatoms are the fused heterocyclic systems possessing wide range of biological activities. In present methodology refluxing para and meta substituted anilines with copper sulphate, methanol, ammonium hydroxide for $10 \mathrm{~h}$ gave 2 -amino substituted benzothiazole which on further refluxing with chloroacetic acid, carbon tetrachloride and potassium carbonate for $15 \mathrm{~h}$ gave 2 -chloro acetylamino benzothiazole followed by reaction with substituted quinolones in presence of dioxane, methyl alcohol produced 2-substituted benzothiazoloquinolates in good yield. The synthesised compounds were purified using column chromatography and characterized using chromatographic and spectrophotometric methods (IR, ${ }^{1}$ HNMR and MS). The synthesized benzothiazoloquinolates were screened for antibacterial activity and were found moderately active against bacterial species.
\end{abstract}

Keywords: Anti-bacterial, Benzothiazole, Disk diffusion and Quinolate.

\section{INTRODUCTION}

In the recent years, most of the population have become vulnerable to bacterial infections causing acute to chronic illness ${ }^{1}$. So the design and development of novel antibacterial agents is essential for the treatment of many forms of bacterial infections. The antibacterial agents act mainly by inhibiting the cell wall synthesis, DNA synthesis and DNA replication (Topoisomerase II or DNA gyrase and Topoisomerase IV), and protein biosynthesis by exhibiting bactericidal and bacteriostatics actions ${ }^{2}$. Heterocyclic moieties are present in nucleic acids, vitamins, proteins and other biomolecules tremendous research novel methodologies have been developed for the synthesis of novel molecules with effective and potent biological components ${ }^{3}$. Benzothiazoles are bicyclic heterocyclic compounds possess fused benzene and thiazoles are leading potential agents with diverse pharmacological properties ${ }^{4}$. A large number of benzothiazole derivatives are used as potent therapeutic agents. Some of the marketed benzothiazole derivatives show antihistaminic $_{8}^{5}$, analgesic ${ }_{9}^{6}$, antihypertensive ${ }^{7}$, antiallergic 8, antiulcer 9, antipsychotic 10, uricosuric agents ${ }^{11}$ and antiviral agents. Quinolones are group of broadspectrum bacteriocides used to treat bacterial infections. Antibacterials containing fluoroquinolone group are the most successful in the field of antimicrobial therapy. Antimicrobial, local anaesthetic, antihelmenthic, pesticidal acitivites of ester compounds already have been reported ${ }^{12}$. In the present investigation 10 compounds (5a$5 \mathrm{j})$ have been synthesised by condensing benzothiazole with fluoroquinolones and converted to their ester forms and evaluated for antibacterial acitivity studies had shown promising results.

\section{MATERIALS AND METHODS}

Chemicals and all solvents of grade AR used in his investigation were procured from Aldrich chemicals, Hychem laboratories. Infrared spectra were recorded on Perkin Elmer Model 
283B instrument and values are given in $\mathrm{cm}^{-1}$, Proton magnetic resonance spectra were recorded on Avance-300 MHz Bruker UX-NMR instrument. The samples were made in $\mathrm{CCl}_{4}$ or chloroform-d $(1: 1)$ or DMSO- $d_{6}$ using tetra methyl silane (TMS) as the internal standard and are given in the $\delta$ scale. Analytical TLC was performed on pre coated silica gel-60 $\mathrm{F}_{254}$ $(0.5 \mathrm{~mm})$ glass plates. Visualization of the spots on TLC plates was achieved by exposure to iodine vapors and ultraviolet light. All solvents used for gel column chromatography were distilled prior to use. Silica gel used was 100-200 mesh \& 60-120 mesh. Cultures of five bacterial strains gram positive (Bacillus subtilis, Bacillus cereus, staphylococcus aureus) and gram negative (Eschericia Coli, Pseudomonas aeruginosa) were used for antibacterial studies and were sub cultured prior to testing.

General methods of synthesis ${ }^{13-14}$

Procedure for synthesis of 2-aminosubstituted benzothiazoles

To a stirring solution of $(0.05 \mathrm{~mol})$ of substituted methyl aniline , (19.43) $\mathrm{g}$ of copper sulphate dissolved in $90 \mathrm{ml}$ of $96 \%$ glacial acetic acid, methanol $(4 \mathrm{ml} 0.05 \mathrm{~mol})$ dissolved in glacial acetic acid $(37.5 \mathrm{ml})$ was added slowly drop wise below $35^{\circ}$. The whole mixture was stirred for $10 \mathrm{~h}$, filtered and residue washed with water. The filtrate and washings were neutralised with ammonium hydroxide, filtered and dried to get 2-amino substituted benzothiazoles.

\section{Procedure for synthesis of 2-(2- chloroacetylamino)-substituted benzothiazoles}

Equimolar solutions of benzothiazole ( 0.01 $\mathrm{mmol})$ and chloroacetic acid $(0.01 \mathrm{mmol})$ in carbon tetrachloride $(30 \mathrm{ml})$ in the presence of $\mathrm{K}_{2} \mathrm{CO}_{3}$ were refluxed at $90^{\circ}$ for $15 \mathrm{~h}$. The product was dried and recrystallised from methanol.

\section{Procedure for synthesis of Benzothiazolo fluorquinolates}

A mixture of benzothiazole $(0.05 \mathrm{mmol})$, quinolone $(0.05 \mathrm{mmol})$ and $\mathrm{NaHCO}_{3}(0.05$ $\mathrm{mmol})$ in dioxane $(10 \mathrm{ml})$, and methanol $(5 \mathrm{ml})$ was heated at $85-90^{\circ}$ for $12 \mathrm{~h}$. Then water $(20 \mathrm{ml})$, was added and the precipitate was filtered and washed with water to give final product.

Schematic Representation of synthetic route was shown in figure. 1 and substituent groups for synthesis of derivatives is shown in table. 1

\section{SPECTRAL DATA}

$5 a$
1-Cyclobutyl -7-(4-(N-3-methyl (5-methyl-1, 3-benzothiazol-2-yl) amino)-2-oxoethyl) piperazin-1-yl)-1, 4-dihydro-4-oxoquinoline-3-carboxylate IR $\left(\mathbf{c m}^{-1}\right)$ 3340 (NH str), 3076-2890 (C-H str), 1715 ( $\mathrm{C}=\mathrm{O} \mathrm{str}), 1666$ (CONH str), 1628 ( $\mathrm{C}=\mathrm{O} \mathrm{str})$, 1528 (C=C str), 1257 (C-O str), 1142 (C-N str).

\section{${ }^{1} \mathrm{H}$ NMR (DMSO-d ${ }_{6}$ ) $\delta \mathrm{ppm}$}

$1.34\left(\mathrm{~m}, 6 \mathrm{H},-\mathrm{CH}_{2} \mathrm{CH}_{2} \mathrm{CH}_{2}\right.$ - cyclobutyl), 3.28$3.84(\mathrm{~m}, 9 \mathrm{H}$, piperazine-H and cyclopropyl-H), $4.16\left(\mathrm{~s}, 2 \mathrm{H},-\mathrm{CH}_{2}\right.$ methylene bridge), 4.84 (s, $1 \mathrm{H},-\mathrm{NH}), 7.02-7.96\left\{\mathrm{~m}, 5 \mathrm{H}\right.$, aromatic $\left(\mathrm{H}_{5}, \mathrm{H}_{8-}\right.$ quinolone and $\mathrm{H}_{4^{\prime}}, \mathrm{H}_{5^{\prime}}, \mathrm{H}_{7}$-benzothiazole)\}, $8.12\left(\mathrm{~s}, 1 \mathrm{H}, \mathrm{H}_{2}\right.$-quinolone), 15.08 (s br, $1 \mathrm{H}$, $\mathrm{COOH}), 2.22\left(3 \mathrm{H}, \mathrm{s}, \mathrm{COCH}_{3}\right)$.

Mass (ESIMS) m/z: $562[\mathrm{M}+1]^{+}$

\section{$5 b$}

1-Cyclobutyl -7-(4-(N-3-methyl (6-chloro, 5methyl-1, 3-benzothiazol-2-yl) amino)-2oxoethyl) piperazin-1-yl)-1, 4-dihydro-4oxo-quinoline-3-carboxylate

IR $\left(\mathrm{cm}^{-1}\right)$ : 3340 (NH str), 3076-2890 (C-H str), 1715 (C=O str), 1666 (CONH str), 1628 ( $\mathrm{C}=\mathrm{O}$ str), 1528 (C=C str), 1257 (C-O str), 1142 (C-N str), 921 ( $\mathrm{C}=\mathrm{O}$ str).

\section{${ }^{1} \mathrm{H}$ NMR (DMSO-d $\mathrm{d}_{6}$ ) $\delta \mathrm{ppm}$}

$1.34\left(\mathrm{~m}, 6 \mathrm{H},-\mathrm{CH}_{2} \mathrm{CH}_{2} \mathrm{CH}_{2}\right.$ - cyclobutyl), 3.28$3.84(\mathrm{~m}, 9 \mathrm{H}$, piperazine- $\mathrm{H}$ and cyclopropyl-H), $4.16\left(\mathrm{~s}, 2 \mathrm{H},-\mathrm{CH}_{2}\right.$ methylene bridge), $4.84(\mathrm{~s}$, $1 \mathrm{H},-\mathrm{NH}), 7.02-7.96\left\{\mathrm{~m}, 5 \mathrm{H}\right.$, aromatic $\left(\mathrm{H}_{5}, \mathrm{H}_{8^{-}}\right.$ quinolone and $\mathrm{H}_{4}, \mathrm{H}_{5^{\prime}}, \mathrm{H}_{7}$-benzothiazole)\}, $8.12\left(\mathrm{~s}, 1 \mathrm{H}, \mathrm{H}_{2}\right.$-quinolone), $2.22(3 \mathrm{H}, \mathrm{s}$, $\left.\mathrm{COCH}_{3}\right)$.

Mass (ESIMS) m/z: $619[\mathrm{M}+\mathrm{Na}]^{+}$

$5 c$

1-Cyclobutyl, 8-methyl-7-(4-(N-(5-methyl-1, 3-benzothiazol-2-yl) amino)-2-oxoethyl) piperazin-1-yl)-1,4-dihydro-4-oxo-quinoline3-carboxylate

IR ( $\mathbf{c m}^{-1}$ ): 3340 (NH str), 3076-2890 (C-H str), 1715 (C=O str), 1666 (CONH str), 1628 ( $\mathrm{C}=\mathrm{O}$ str), 1528 (C=C str), 1257 (C-O str), 1142 (C-N str).

\section{${ }^{1} \mathrm{H}$ NMR (DMSO-d $\mathrm{d}_{6}$ ) $\delta \mathrm{ppm}$}

$1.34\left(\mathrm{~m}, 6 \mathrm{H},-\mathrm{CH}_{2} \mathrm{CH}_{2} \mathrm{CH}_{2}\right.$ - cyclobutyl), 3.28$3.84(\mathrm{~m}, 9 \mathrm{H}$, piperazine- $\mathrm{H}$ and cyclopropyl- $\mathrm{H})$, $4.16\left(\mathrm{~s}, 2 \mathrm{H},-\mathrm{CH}_{2}\right.$ methylene bridge), $4.84(\mathrm{~s}$, $1 \mathrm{H},-\mathrm{NH}), 7.02-7.96\left\{\mathrm{~m}, 5 \mathrm{H}\right.$, aromatic $\left(\mathrm{H}_{5}, \mathrm{H}_{8^{-}}\right.$ quinolone and $\mathrm{H}_{4}, \mathrm{H}_{5^{\prime}}, \mathrm{H}_{7}$-benzothiazole)\}, $8.12\left(\mathrm{~s}, 1 \mathrm{H}, \mathrm{H}_{2}\right.$-quinolone), $2.22(3 \mathrm{H}, \mathrm{s}$, $\left.\mathrm{COCH}_{3}\right)$.

Mass (ESIMS) m/z: $562[\mathrm{M}+1]^{+}$ 
5d

1-ethyl, 8-methoxy -7-(4-(N-(5-methyl-1, 3benzothiazol-2-yl) piperazin-1-yl)-1, amino)-2-oxoethyl) quinoline-3-carboxylate IR $\left(\mathrm{cm}^{-1}\right)$

3340 (NH str), 3076-2890 (C-H str), 1715 ( $\mathrm{C}=\mathrm{O}$ str), 1666 (CONH str), 1628 ( $\mathrm{C}=\mathrm{O}$ str), 1528 (C=C str), 1257 (C-O str), 1142 (C-N str).

${ }^{1} \mathrm{H}$ NMR (DMSO-d ${ }_{6}$ ) $\delta$ ppm

1-2.8 (m, 5H, - $\mathrm{CH}_{2}-\mathrm{CH}_{3}$-ethyl), 3.28-3.84 (m, $9 \mathrm{H}$, piperazine- $\mathrm{H}$ and cyclopropyl-H), 4.16 (s, $2 \mathrm{H},-\mathrm{CH}_{2}$ methylene bridge), $4.84(\mathrm{~s}, 1 \mathrm{H},-\mathrm{NH})$, 7.02-7.96 $\left\{\mathrm{m}, 5 \mathrm{H}\right.$, aromatic $\left(\mathrm{H}_{5}, \mathrm{H}_{8}\right.$-quinolone and $\mathrm{H}_{4^{\prime}}, \mathrm{H}_{5^{\prime}}, \mathrm{H}_{7}{ }^{\prime}$-benzothiazole) $\}, 8.12(\mathrm{~s}, 1 \mathrm{H}$, $\mathrm{H}_{2}$-quinolone), $2.22\left(3 \mathrm{H}, \mathrm{s}, \mathrm{COCH}_{3}\right)$.

Mass (ESIMS) m/z: $574[\mathrm{M}+\mathrm{Na}]^{+}$

$5 e$

1-ethyl, 8-methoxy -7-(4-(N-(6-chloro, 5methyl-1, 3-benzothiazol-2-yl) amino)-2oxoethyl) piperazin-1-yl)-1, 4-dihydro-4oxo-quinoline-3-carboxylate

IR $\left(\mathbf{c m}^{-1}\right)$

3340 (NH str), 3076-2890 (C-H str), 1715 ( $\mathrm{C}=\mathrm{O} \mathrm{str}), 1666$ (CONH str), 1628 ( $\mathrm{C}=\mathrm{O} \mathrm{str})$, 1528 (C=C str), 1257 (C-O str), 1142 (C-N str), 921( $\mathrm{C}=\mathrm{O}$ str).

${ }^{1} \mathrm{H}$ NMR (DMSO-d 6 ) $\delta$ ppm

1-2.8 (m, $5 \mathrm{H},-\mathrm{CH}_{2}-\mathrm{CH}_{3}$-ethyl), 3.28-3.84 (m, $9 \mathrm{H}$, piperazine- $\mathrm{H}$ and cyclopropyl-H), $4.16(\mathrm{~s}$, $2 \mathrm{H},-\mathrm{CH}_{2}$ methylene bridge), $4.84(\mathrm{~s}, 1 \mathrm{H},-\mathrm{NH})$, 7.02-7.96 $\left\{\mathrm{m}, 5 \mathrm{H}\right.$, aromatic $\left(\mathrm{H}_{5}, \mathrm{H}_{8}\right.$-quinolone and $\mathrm{H}_{4}, \mathrm{H}_{5^{\prime}}, \mathrm{H}_{7}$-benzothiazole) $\}, 8.12(\mathrm{~s}, 1 \mathrm{H}$, $\mathrm{H}_{2}$-quinolone), $2.22\left(3 \mathrm{H}, \mathrm{s}, \mathrm{COCH}_{3}\right)$.

Mass (ESIMS) m/z: $587[\mathrm{M}+1]^{+}$

$5 f$

1-propyl, 8-methoxy -7-(4-(N-(6-chloro, 5methyl-1, 3-benzothiazol-2-yl) amino)-2oxoethyl) piperazin-1-yl)-1,4-dihydro-4-oxoquinoline-3-carboxylate

IR ( $\left.\mathrm{cm}^{-1}\right)$ : 3340 (NH str), 3076-2890 (C-H str), 1715 (C=O str), 1666 (CONH str), 1628 ( $\mathrm{C}=\mathrm{O}$ str), 1528 (C=C str), 1257 (C-O str), 1142 (C-N str), 921 ( $\mathrm{C}=\mathrm{O}$ str).

${ }^{1} \mathrm{H}$ NMR (DMSO-d ${ }_{6}$ ) $\delta$ ppm

1-2.8 (m, 7H, - $\mathrm{CH}_{2} \mathrm{CH}_{2} \mathrm{CH}_{3}$ - propyl), 3.28-3.84 $(\mathrm{m}, 9 \mathrm{H}$, piperazine- $\mathrm{H}$ and cyclopropyl- $\mathrm{H}), 4.16$ (s, $2 \mathrm{H},-\mathrm{CH}_{2}$ methylene bridge), $4.84(\mathrm{~s}, 1 \mathrm{H}$, $\mathrm{NH})$, 7.02-7.96 $\left\{\mathrm{m}, 5 \mathrm{H}\right.$, aromatic $\left(\mathrm{H}_{5}, \mathrm{H}_{8-}\right.$ quinolone and $\mathrm{H}_{4}, \mathrm{H}_{5^{\prime}}, \mathrm{H}_{7}$-benzothiazole)\}, $8.12\left(\mathrm{~s}, 1 \mathrm{H}, \mathrm{H}_{2}\right.$-quinolone $), 2.22(3 \mathrm{H}, \mathrm{s}$, $\left.\mathrm{COCH}_{3}\right)$.
Mass (ESIMS) m/z: $623[\mathrm{M}+\mathrm{Na}]^{+}$

$5 \mathrm{~g}$

1-ethyl, 8-methoxy -7-(4-(3-chloro-(N-(5methyl-1, 3-benzothiazol-2-yl) amino)-2oxoethyl) piperazin-1-yl)-1,4-dihydro-4-oxoquinoline-3-carboxylate

IR ( $\left.\mathrm{cm}^{-1}\right)$ : 3340 (NH str), 3076-2890 (C-H str), 1715 (C=O str), 1666 (CONH str), 1628 ( $\mathrm{C}=\mathrm{O}$ str), 1528 (C=C str), 1257 (C-O str), 1142 (C-N str).

\section{${ }^{1} \mathrm{H}$ NMR (DMSO-d $\mathrm{d}_{6}$ ) $\delta \mathrm{ppm}$}

1-2.8 (m, 5H, - $\mathrm{CH}_{2} \mathrm{CH}_{3}$ - ethyl), 3.28-3.84 (m, $9 \mathrm{H}$, piperazine-H and cyclopropyl-H), $4.16(\mathrm{~s}$, $2 \mathrm{H},-\mathrm{CH}_{2}$ methylene bridge), $4.84(\mathrm{~s}, 1 \mathrm{H},-\mathrm{NH})$, 7.02-7.96 $\left\{\mathrm{m}, 5 \mathrm{H}\right.$, aromatic $\left(\mathrm{H}_{5}, \mathrm{H}_{8}\right.$-quinolone and $\mathrm{H}_{4^{\prime}}, \mathrm{H}_{5^{\prime}}, \mathrm{H}_{7}{ }^{\prime}$-benzothiazole)\}, $8.12(\mathrm{~s}, 1 \mathrm{H}$, $\mathrm{H}_{2}$-quinolone), $2.22\left(3 \mathrm{H}, \mathrm{s}, \mathrm{COCH}_{3}\right)$.

Mass (ESIMS) m/z: $587[\mathrm{M}+1]^{+}$

$5 \mathrm{~h}$

1-propyl, 8-methoxy -7-(4-(3-chloro-(N-(6chloro, 5-methyl-1, 3-benzothiazol-2-yl) amino)-2-oxoethyl) piperazin-1-yl)-1, 4dihydro-4-oxo-quinoline-3-carboxylate IR $\left(\mathrm{cm}^{-1}\right)$ : 3340 (NH str), 3076-2890 (C-H str), 1715 ( $\mathrm{C}=\mathrm{O}$ str), 1666 (CONH str), 1628 ( $\mathrm{C}=\mathrm{O}$ str), 1528 ( $\mathrm{C}=\mathrm{C}$ str), 1257 (C-O str), 1142 (C-N str), 921 ( $\mathrm{C}=\mathrm{O}$ str).

\section{${ }^{1} \mathrm{H}$ NMR (DMSO-d $\left.\mathrm{d}_{6}\right) \delta \mathrm{ppm}$}

1-2.8 (m, 7H, - $\mathrm{CH}_{2} \mathrm{CH}_{2} \mathrm{CH}_{3}$ - propyl), 3.28-3.84 ( $\mathrm{m}, 9 \mathrm{H}$, piperazine-H and cyclopropyl-H), 4.16 (s, $2 \mathrm{H},-\mathrm{CH}_{2}$ methylene bridge), $4.84(\mathrm{~s}, 1 \mathrm{H},-$ $\mathrm{NH}),{ }^{7.02-7.96}\left\{\mathrm{~m}, 5 \mathrm{H}\right.$, aromatic $\left(\mathrm{H}_{5}, \mathrm{H}_{8^{-}}\right.$ quinolone and $\mathrm{H}_{4^{\prime}}, \mathrm{H}_{5^{\prime}}, \mathrm{H}_{7}{ }^{\prime}$-benzothiazole)\}, $8.12\left(\mathrm{~s}, 1 \mathrm{H}, \mathrm{H}_{2}\right.$-quinolone $), 2.22(3 \mathrm{H}, \mathrm{s}$, $\mathrm{COCH}_{3}$ ).

Mass (ESIMS) m/z: $658[\mathrm{M}+\mathrm{Na}]^{+}$

$5 \mathbf{i}$

1-propyl, 8-methoxy -7-(4-(3-chloro-(N-(5methyl-1, 3-benzothiazol-2-yl) amino)-2oxoethyl) piperazin-1-yl)-1, 4-dihydro-4oxo-quinoline-3-carboxylate

IR $\left(\mathbf{c m}^{-1}\right)$

3340 (NH str), 3076-2890 (C-H str), 1715 ( $\mathrm{C}=\mathrm{O} \mathrm{str}), 1666$ (CONH str), 1628 ( $\mathrm{C}=\mathrm{O} \mathrm{str})$, 1528 (C=C str), 1257 (C-O str), 1142 (C-N str);

${ }^{1} \mathrm{H}$ NMR (DMSO-d ${ }_{6}$ ) $\delta$ ppm

1-2.8 (m, 7H, - $\mathrm{CH}_{2} \mathrm{CH}_{2} \mathrm{CH}_{3}$ - propyl), 3.28-3.84 $(\mathrm{m}, 9 \mathrm{H}$, piperazine- $\mathrm{H}$ and cyclopropyl- $\mathrm{H}), 4.16$ (s, $2 \mathrm{H},-\mathrm{CH}_{2}$ methylene bridge), $4.84(\mathrm{~s}, 1 \mathrm{H},-$ $\mathrm{NH})$, 7.02-7.96 $\left\{\mathrm{m}, 5 \mathrm{H}\right.$, aromatic $\left(\mathrm{H}_{5}, \mathrm{H}_{8^{-}}\right.$ quinolone and $\mathrm{H}_{4^{\prime}}, \mathrm{H}_{5^{\prime}}, \mathrm{H}_{7}{ }^{\prime}$-benzothiazole)\}, 
$8.12\left(\mathrm{~s}, 1 \mathrm{H}, \mathrm{H}_{2}\right.$-quinolone), $2.22(3 \mathrm{H}, \mathrm{s}$, $\left.\mathrm{COCH}_{3}\right)$.

Mass (ESIMS) m/z: 601[M+1] ${ }^{+}$

$5 j$

1-ethyl, 8-methyl -7-(4-(N-(6-chloro, 5methyl-1, 3-benzothiazol-2-yl) amino)-2oxoethyl) piperazin-1-yl)-1, 4-dihydro-4oxo-quinoline-3-carboxylate

IR $\left(\mathrm{cm}^{-1}\right)$ : 3340 (NH str), 3076-2890 (C-H str), 1715 (C=O str), 1666 (CONH str), 1628 ( $\mathrm{C}=\mathrm{O}$ str), 1528 (C=C str), 1257 (C-O str), 1142 (C-N str),

\section{${ }^{1} \mathrm{H}$ NMR (DMSO-d $\left.\mathrm{d}_{6}\right) \delta \mathrm{ppm}$}

1-2.8 (m, 4H, $-\mathrm{CH}_{2} \mathrm{CH}_{3}$ - ethyl), 3.28-3.84 (m, $9 \mathrm{H}$, piperazine- $\mathrm{H}$ and cyclopropyl- $\mathrm{H}), 4.16(\mathrm{~s}$, $2 \mathrm{H},-\mathrm{CH}_{2}$ methylene bridge), $4.84(\mathrm{~s}, 1 \mathrm{H},-\mathrm{NH})$, 7.02-7.96 $\left\{\mathrm{m}, 5 \mathrm{H}\right.$, aromatic $\left(\mathrm{H}_{5}, \mathrm{H}_{8}\right.$-quinolone and $\mathrm{H}_{4^{\prime}}, \mathrm{H}_{5^{\prime}}, \mathrm{H}_{7}$-'benzothiazole)\}, 8.12 (s, $1 \mathrm{H}$, $\mathrm{H}_{2}$-quinolone), ), $2.22\left(3 \mathrm{H}, \mathrm{s}, \mathrm{COCH}_{3}\right.$ ).

Mass (ESIMS) m/z: $609[\mathrm{M}+\mathrm{Na}]^{+}$

\section{ANTIBACTERIAL ACTIVITY ${ }^{15}$}

The synthesised compounds were screened for their biological activity studies using five different strains of gram positive (Bacillus subtilis, Bacillus cereus, staphylococcus aureus) and gram negative (Eschericia Coli, Pseudomonas aeruginosa) bacterial species.

\section{RESULTS AND DISCUSSION}

In the present investigation, we have synthesised the series of benzothiazole substituted quinolates in a three step procedure using appropriate reagents, isolated and purified using column chromatography, characterised using thin layer chromatography, Infra red spectroscopy, ${ }^{1}$ HNMR and Mass spectroscopy. The reaction conditions were optimised to achieve the compounds in a better yield which was shown in table. 2 . The synthesised compounds were evaluated for antibacterial pharmacological activity. The experimental results revealed that the compounds possessing electron withdrawing substituents possessed satisfactory antibacterial activity compared to the standard antibacterial drugs amoxicillin and ciprofloxacin.

\section{CONCLUSION}

From the present methodology, 2-substituted benzothiazoloquinolate derivatives can be synthesised in good yield from substituted anilines by optimising reaction conditions (varying solvents and temperature conditions). In future, synthesised derivatives may serve as important precursors for the development of novel compounds with potent antibacterial activity.

\section{ACKNOWLEDEMENTS}

The authors are thankful to AICTE and Rajiv Gandhi University of Health sciences, Bangalore for financial support to carry out these investigative studies.<smiles></smiles>

Fig.1 (Scheme): Synthesis of 2-substituted benzothiazolo quinolates (5a-j) 1. Substituted methyl aniline 2. 2-amino-substituted benzothiazoles 


\section{2-(2-chloroacetylamino)-substituted benzothiazole}

4. Quinolone 5. Benzothiazolo quinolate

Table 1: Substituent groups for the synthesis of novel benzothiazoloquinolates

\begin{tabular}{|c|c|c|c|c|c|}
\hline Compd & $\mathbf{R}$ & $\mathbf{R}^{1}$ & $\mathbf{R}^{2}$ & $\mathbf{R}^{3}$ & $\mathbf{M F}$ \\
\hline $5 \mathrm{a}$ & $-\mathrm{H}$ & - & $-\mathrm{H}$ & $-\mathrm{CH}_{3}$ & $\mathrm{C}_{30} \mathrm{H}_{35} \mathrm{~N}_{5} \mathrm{O}_{4} \mathrm{~S}$ \\
\hline $5 \mathrm{~b}$ & $-\mathrm{Cl}$ & - & $-\mathrm{H}$ & $-\mathrm{CH}_{3}$ & $\mathrm{C}_{30} \mathrm{H}_{34} \mathrm{CIN}_{5} \mathrm{O}_{4} \mathrm{~S}$ \\
\hline $5 \mathrm{c}$ & $-\mathrm{H}$ & - & $-\mathrm{CH}_{3}$ & $-\mathrm{H}$ & $\mathrm{C}_{30} \mathrm{H}_{35} \mathrm{~N}_{5} \mathrm{O}_{4} \mathrm{~S}$ \\
\hline $5 \mathrm{~d}$ & $-\mathrm{H}$ & $-\mathrm{CH}_{2} \mathrm{CH}_{3}$ & $-\mathrm{OCH}_{3}$ & $-\mathrm{H}$ & $\mathrm{C}_{28} \mathrm{H}_{33} \mathrm{~N}_{5} \mathrm{O}_{5} \mathrm{~S}$ \\
\hline $5 \mathrm{e}$ & $-\mathrm{Cl}$ & $-\mathrm{CH}_{2} \mathrm{CH}_{3}$ & $-\mathrm{OCH}_{3}$ & $-\mathrm{H}$ & $\mathrm{C}_{28} \mathrm{H}_{32} \mathrm{CIN}_{5} \mathrm{O}_{5} \mathrm{~S}$ \\
\hline $5 \mathrm{f}$ & $-\mathrm{Cl}$ & $-\mathrm{CH}_{2} \mathrm{CH}_{2} \mathrm{CH}_{3}$ & $-\mathrm{OCH}_{3}$ & $-\mathrm{H}$ & $\mathrm{C}_{29} \mathrm{H}_{34} \mathrm{CIN}_{5} \mathrm{O}_{5} \mathrm{~S}$ \\
\hline $5 \mathrm{~g}$ & $-\mathrm{H}$ & $-\mathrm{CH}_{2} \mathrm{CH}_{3}$ & $-\mathrm{OCH}_{3}$ & $-\mathrm{Cl}$ & $\mathrm{C}_{28} \mathrm{H}_{32} \mathrm{CIN}_{5} \mathrm{O}_{5} \mathrm{~S}$ \\
\hline $5 \mathrm{~h}$ & $-\mathrm{Cl}$ & $-\mathrm{CH}_{2} \mathrm{CH}_{2} \mathrm{CH}_{3}$ & $-\mathrm{OCH}_{3}$ & $-\mathrm{Cl}$ & $\mathrm{C}_{29} \mathrm{H}_{33} \mathrm{Cl}_{2} \mathrm{~N}_{5} \mathrm{O}_{5} \mathrm{~S}$ \\
\hline $5 \mathrm{i}$ & $-\mathrm{H}$ & $-\mathrm{CH}_{2} \mathrm{CH}_{2} \mathrm{CH}_{3}$ & $-\mathrm{OCH}_{3}$ & $-\mathrm{Cl}$ & $\mathrm{C}_{29} \mathrm{H}_{34} \mathrm{CIN}_{5} \mathrm{O}_{5} \mathrm{~S}$ \\
\hline $5 \mathrm{j}$ & $-\mathrm{Cl}$ & $-\mathrm{CH}_{2} \mathrm{CH}_{3}$ & $-\mathrm{CH}_{3}$ & $-\mathrm{H}$ & $\mathrm{C}_{28} \mathrm{H}_{32} \mathrm{CIN}_{5} \mathrm{O}_{4} \mathrm{~S}$ \\
\hline
\end{tabular}

Table 2: Optimization of reaction conditions<smiles>[R]c1cc(N)ccc1C</smiles>

Reflux 10-15h<smiles>[X]C(=O)CNc1nc2cc([R])c(C)cc2s1</smiles>

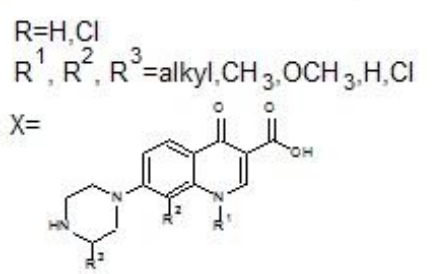

\begin{tabular}{|c|c|c|c|c|}
\hline Entry & Solvent $^{\mathbf{a}}$ & Temp $\left.^{\mathbf{0}} \mathbf{C}\right)^{\mathbf{b}}$ & Time(h) $^{\mathbf{c}}$ & Yield (\%) $^{\mathbf{d}}$ \\
\hline 1 & $\mathrm{CCl}_{4}$ & 90 & $10-15$ & 75 \\
\hline 2 & Dioxane & 90 & $10-15$ & 70 \\
\hline 3 & Hexane & 90 & $10-15$ & 60 \\
\hline 4 & EtOAc & 90 & $10-15$ & 55 \\
\hline 5 & $\mathrm{CH}_{3} \mathrm{CN}$ & 90 & $10-15$ & 60 \\
\hline 6 & Benzene & 90 & $10-15$ & 55 \\
\hline 7 & $\mathrm{CHCl}_{3}$ & 90 & $10-15$ & 45 \\
\hline 8 & $\mathrm{THF}$ & 90 & $10-15$ & 55 \\
\hline 9 & Toluene & 90 & $10-15$ & 60 \\
\hline 10 & DCM & 90 & $10-15$ & 65 \\
\hline
\end{tabular}

a. Solvent system $b$. Temperature conditions

c. Time provided to complete the reaction

d. Isolated yield of the product

\section{REFERENCES}

1. Jubie $S$, Kumar $R$ and Yellareddy. Microwave assisted synthesis of some novel benzimidazole substituted fluoroquinolones and their antimicrobial evaluation. Pharm Sci and Res. 2010;2(2):69-76.

2. Rueping $M$, Stoeckel $M$, Sugiono $E$ and Theissmann T. Asymmetric metalfree synthesis of fluoroquinolones by organocatalytic hydrogenation.
Tetrahedron .2010; 66(33): 65656568.

3. Wang $\mathrm{X}$, Xiaotong $\mathrm{L}$ and Renhe $\mathrm{H}$. Elemental Sulfur-Mediated Decarboxylative Redox Cyclization Reaction: Copper-Catalyzed Synthesis of 2-Substituted Benzothiazoles. Synlett. 2018;29(2):219-24.

4. Sun $Y$, Jiang $H, W u W$, Zeng $W$ and Wu X. Copper-Catalyzed Synthesis of Substituted Benzothiazoles via Condensation of 2- 
Aminobenzenethiols with Nitriles.Org Lett. 2013; 15(7):1598-1601.

5. Dubar $F$, Wintjens $R$ and Erica $S$. Ester prodrugs of ciprofloxacin as DNAgyrase inhibitors: synthesis, anti parasitic evaluation and docking studies. Med Chem Commun. 2011;2:430-35.

6. ZhaoYang Z, Renhe $\mathrm{Hu}$ and Xiaotong L. One-pot copper-catalyzed synthesis of 2-substituted benzothiazoles from 2-iodoanilines, benzyl chlorides and elemental sulfur. Tetrahedron Letters .2017;58(24):2366-69.

7. Chen Y, Fang K, Sheu J, Hsu S and Tzeng C. Synthesis and Antibacterial Evaluation of Certain Quinolone Derivatives. $J$ Med Chem. 2001;44(14):2374-77.

8. Chai Y, Wan Z and Liu B. Synthesis and in vitro antibacterial activity of 7(4-alkoxyimino-3-amino-3methylpiperidin-1-yl) fluoroquinolone derivatives. European Journal of Medicinal Chemistry. 2009;44(10):4063-69.

9. Lima D, Penteado $F$ and Vieira $M$. a-Keto Acids as Acylating Agents in the Synthesis of 2-Substituted Benzothiazoles and Benzoselenazoles. EURJOC. 2017;26:3830-36.

10. Reddy F, Clemence R, Burger L, Hoegy $F$, Isabelle $M$ and Schalk $S$.
Synthesis of pyochelin-norfloxacin conjugates. Bioorganic \& Medicinal Chemistry Letters. 2007;17(3):640-44.

11. Dixit S, Mishra $N$ and Sharma $M$. Synthesis and in vitro antiplasmodial activities of fluoroquinolone analogs. European Journal of Medicinal Chemistry. 2012;51:52-59.

12. Hiari Y, Mazari I, Shakya A, Darwish $R$ and Dahab R. Synthesis and Antibacterial Properties of New 8Nitrofluoro quinolone Derivatives. Molecules. 2007;12(6):1240-58.

13. Sharma $P$, Kumar $R$, Chaudhary $M$, Sharma A and Rajak H. Synthesis and biological evaluation of novel benzothiazole clubbed fluoroquinolone derivatives. Journal of Enzyme Inhibition and Medicinal Chemistry.2013;28(1):1-10.

14. Anquetin G, and Mahmoudi N. Synthesis of new fluoroquinolones and evaluation of their in vitro activity on Toxoplasmagondii and Plasmodiu $m$ spp. Bioorganic \& Medicinal Chemistry Letters. 2004;14(11):277376.

15. Khokra S, Kanika Arora K, Mehta H, Aggarwal A and Yadav M. Common methods to synthesise benzothiazole derivatives and their medicinal significance A review IJPSR. $2011 ; 2(6): 1356-77$. 